\title{
Tonic Neck Reflex
}

National Cancer Institute

\section{Source}

National Cancer Institute. Tonic Neck Reflex. NCI Thesaurus. Code C81324.

An involuntary, primal response in the neonate in which the arm extends to follow the infant's gaze, while the opposing arm and leg flex inward. 